\title{
Polarimetric parity-time symmetry in a photonic system
}

\author{
Lingzhi Li ${ }^{1}$, Yuan Cao ${ }^{1}$, Yanyan Zhi', Jiejun Zhang $\mathbb{D}^{1}$, Yuting Zou', Xinhuan Feng ${ }^{1}$, Bai-Ou Guan ${ }^{1}$ and Jianping Yao (1) ${ }^{1,2}$
}

\begin{abstract}
Parity-time (PT) symmetry has attracted intensive research interest in recent years. PT symmetry is conventionally implemented between two spatially distributed subspaces with identical localized eigenfrequencies and complementary gain and loss coefficients. The implementation is complicated. In this paper, we propose and demonstrate that PT symmetry can be implemented between two subspaces in a single spatial unit based on optical polarimetric diversity. By controlling the polarization states of light in the single spatial unit, the localized eigenfrequencies, gain, loss, and coupling coefficients of two polarimetric loops can be tuned, leading to PT symmetry breaking. As a demonstration, a fiber ring laser based on this concept supporting stable and single-mode lasing without using an ultranarrow bandpass filter is implemented.
\end{abstract}

\section{Introduction}

A parity-time (PT) symmetric system is a special nonHermitian system of which its Hamiltonian possesses real eigenvalues. Recently, PT symmetry has been investigated extensively in photonic ${ }^{1-13}$ and optoelectronic ${ }^{14,15}$ systems, one of the main reasons being its effectiveness for mode selection in an optical or optoelectronic system ${ }^{11-}$ ${ }^{15}$. For a high-performance continuous-wave laser, singlemode operation is one of the fundamental requirements because it determines the coherence and power stability of the laser output ${ }^{5,6,16}$. For a long-cavity laser, for which it is preferable to have low phase noise, a narrow linewidth, and high optical power, single-mode lasing is challenging due to the small mode spacing ${ }^{5,6,16-24}$. Since the gain spectrum of a gain medium in a laser cavity is usually several orders of magnitude broader than the mode spacing of the laser, the round-trip gain difference between adjacent modes is small, making it difficult to achieve single-mode oscillation by manipulating the lasing

Correspondence: Jiejun Zhang (zhangjiejun@jnu.edu.cn) or Jianping Yao (jpyao@uottawa.ca)

${ }^{1}$ Guangdong Provincial Key Laboratory of Optical Fiber Sensing and Communications, Institute of Photonics Technology, Jinan University, Guangzhou 511442, China

${ }^{2}$ Microwave Photonics Research Laboratory, School of Electrical Engineering and Computer Science, University of Ottawa, Ottawa, ON K1N 6N5, Canada threshold ${ }^{5,6}$. An optical filter can be incorporated into a laser cavity to reduce the number of modes above the threshold. If the optical filter has a sufficiently narrow passband, single-mode lasing can be achieved ${ }^{23,24}$. However, for a long-cavity laser such as a fiber laser with a cavity length on the order of tens of meters, a high- $\mathrm{Q}$ optical filter is needed, making the system costly and endowing it with poor stability ${ }^{25,26}$.

Parity-time symmetry has been proven to be an effective solution for achieving mode selection in a photonic system $^{1-15}$. Conventionally, a PT-symmetric system is implemented between two cross-coupled and spatially distributed optical subspaces, which are engineered to have an identical geometry with complementary gain and loss coefficients. A PT-symmetric system has a strongly enhanced gain difference between the dominant mode and the side modes, thus making single-mode oscillation possible $^{5,6,14,15}$. However, such a system requires two spatially distributed subspaces, which leads to increased structural complexity, high cost, and strong susceptibility to environmental perturbations. Recently, we demonstrated a new type of PT-symmetric system that is realized in the parameter space of optical wavelength, where the spatial duplicity in a conventional PT-symmetric system is eliminated ${ }^{27}$. Since two wavelengths are employed, the 
concept is not applicable to a system where only a single wavelength is supported.

In this paper, we propose and demonstrate that a PTsymmetric system can be implemented in a single spatial unit based on polarimetric diversity, which supports singlewavelength operation. By controlling the polarization states of light in a single spatial unit, the localized eigenfrequencies, gain, loss, and coupling coefficients can be controlled to achieve PT symmetry breaking. As a demonstration, a long-cavity fiber ring laser supporting singlemode lasing without using a high-Q optical filter is implemented. In the experiment, the fiber ring laser has a cavity length of $41.0 \mathrm{~m}$ with a mode spacing as small as $4.88 \mathrm{MHz}$. The employment of polarimetric PT symmetry enables effective suppression of the sidemodes with a suppression ratio greater than $47.9 \mathrm{~dB}$. The linewidth of the light generated by the fiber ring laser is measured to be $129 \mathrm{kHz}$ with a wavelength tunable range of $35 \mathrm{~nm}$. With active stabilization, the linewidth can be reduced to its Lorentzian linewidth of $2.4 \mathrm{kHz}$. The proposed polarimetric PT symmetry concept opens new avenues for the implementation of nonHermitian photonic systems with increased functionalities.

\section{Results}

Figure 1 shows a schematic diagram of the proposed polarimetric PT-symmetric photonic system consisting of two polarimetric loops. The two polarimetric loops, with independently adjustable eigenfrequencies and round-trip gain coefficients, are implemented based on polarimetric diversity in a single physical loop. Specifically, the birefringent path in the fiber ring laser loop creates two polarimetric loops, and the coupled path allows coupling between the two polarimetric loops. Two polarization controllers (PCs), in conjunction with two polarizers (Pol. 1 and Pol. 2), are used to tune the gain and loss coefficients of the eigenvalues in the two polarimetric loops to achieve PT symmetry. As the gain and loss coefficients are increased to values greater than the coupling coefficient, PT symmetry breaking occurs. The two polarimetric loops form a PTsymmetric fiber ring laser with one polarimetric loop supporting the gain modes and the other supporting the loss modes. The polarimetric PT symmetry then strongly enhances the gain difference between the dominant mode and the sidemodes, making single-mode lasing possible without using an ultranarrow bandpass filter.

As shown in Fig. 1, the photonic system has a single unidirectional physical fiber loop that supports two polarimetric loops. An erbium-doped fiber amplifier (EDFA) is incorporated to provide an optical gain. The polarimetric diversity is implemented by controlling the polarization states of light in the fiber loop. The dominant mode can be coarsely chosen with a tunable optical filter (TOF). The output of the system is derived using a 3-dB optical coupler (OC), which is sent to an optical spectrum analyzer (OSA) for spectrum analysis, an optical homodyne system for mode analysis, and an optical selfheterodyne system for linewidth measurement.

A three-paddle fiber-optic PC has a sandwiched structure with a half-wave plate located between two quarterwave plates. The transfer function of a three-paddle PC is given by $^{28}$

$$
F_{P C}=\left[\begin{array}{ll}
\exp \left(i \phi_{2}\right) & 0 \\
0 & 1
\end{array}\right] \times\left[\begin{array}{cc}
\cos \theta & -\sin \theta \\
\sin \theta & \cos \theta
\end{array}\right] \times\left[\begin{array}{ll}
\exp \left(i \phi_{1}\right) & 0 \\
0 & 1
\end{array}\right]
$$

where $\phi_{1}$ and $\phi_{2}$ are the phase retardances introduced to the two orthogonally polarized light waves by the two

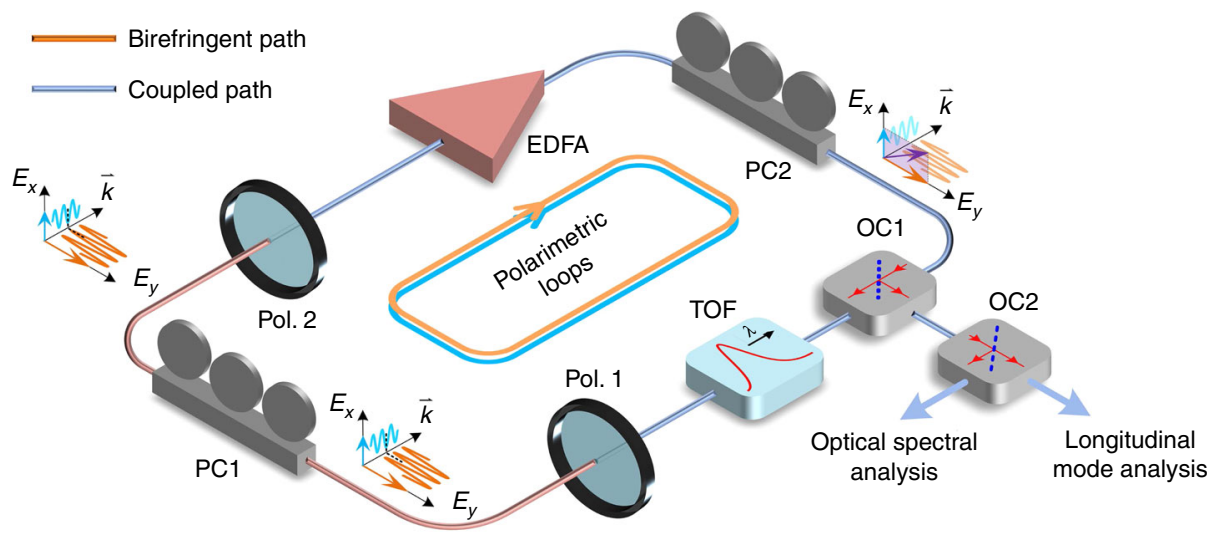

Fig. 1 Schematic diagram of the proposed polarimetric PT-symmetric photonic system. The system consists of a single spatial loop, in which two equivalent polarimetric loops are formed by recirculating light waves of orthogonal polarization states in the loop. To achieve PT symmetry, the phase retardance, power ratio, and coupling coefficient between the orthogonally polarized light waves are tuned by controlling PC1 in the birefringent path, and the lasing threshold is tuned by controlling PC2 in the coupled path. PC: polarization controller; Pol.: polarizer; EDFA: erbiumdoped fiber amplifier; OC: optical coupler; TOF: tunable optical filter 
Table 1 The critical angles that are tuned to achieve polarimetric PT symmetry

\begin{tabular}{llll}
\hline Angle & Definition & Functionality & Tuning method \\
\hline$\theta_{r}$ & $\begin{array}{l}\text { Rotation angle of quarter-wave plate to tune } \\
\text { polarization phase retardance }\end{array}$ & $\begin{array}{l}\text { PT symmetry of the real part of the eigenfrequency of the } \\
\text { polarimetric loops }\end{array}$ & Quarter-wave plate of PC1 \\
$\theta_{i}$ & Optical axis rotation angle from Pol. 1 to Pol. 2 & $\begin{array}{l}\text { PT symmetry of the imaginary part of the eigenfrequency of } \\
\text { the polarimetric loops }\end{array}$ & Half-wave plate of PC1 \\
$\theta_{t}$ & Optical axis rotation angle from Pol. 2 to Pol. 1 & Lasing threshold & Half-wave plate of PC2 \\
\hline
\end{tabular}
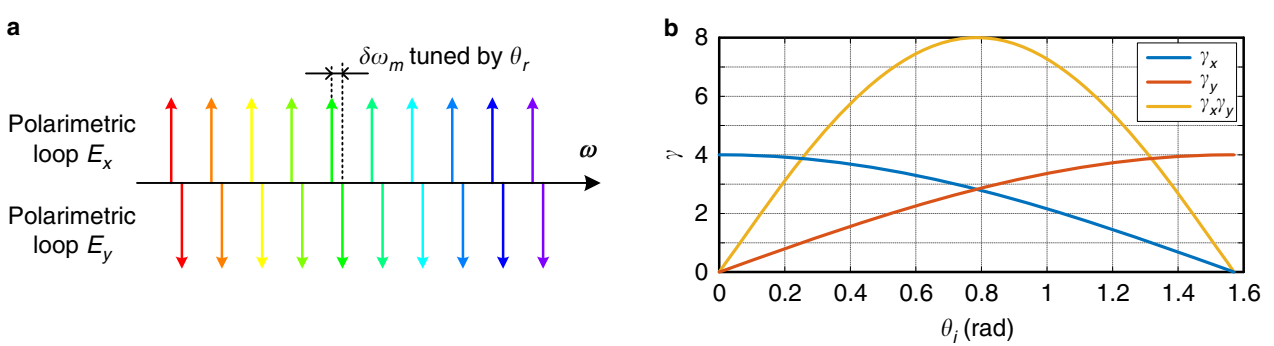

c

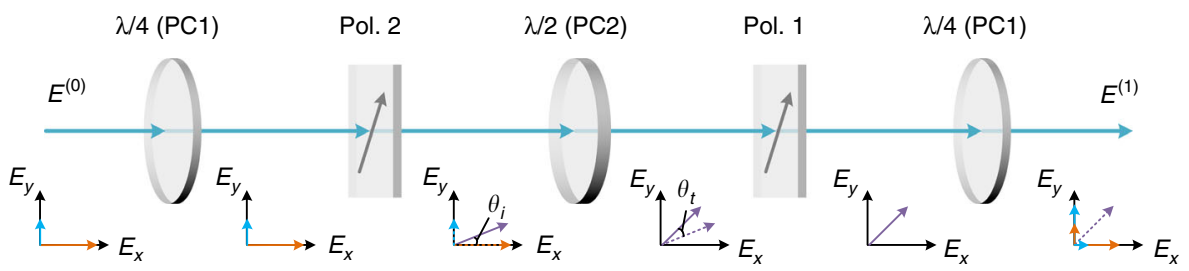

Fig. 2 Polarimetric PT symmetry achieved by controlling the polarizations by tuning PC1 and PC2 in the photonic system. a Compensation for eigenfrequency separation between the polarimetric loops when the phase retardances between $E_{x}$ and $E_{y}$ are tuned by the quarter-wave plate of $\mathrm{PC} 1 ; \mathbf{b}$ round-trip gain and loss coefficient variations of the polarimetric loops when the polarization directions of $E_{x}$ and $E_{y}$ are tuned by the halfwaveplate of $\mathrm{PC}$; and $\mathbf{c}$ illustration of the gain, loss and coupling of the polarization components as light at PC1 propagates through Pol. 1, PC2, and Pol. 2 and then returns to PC1. Part of the polarization component $E_{x}$ is coupled to the other polarization component $E_{y}$ and vice versa

quarter-wave plates and $\theta$ is the rotation angle of the polarization direction introduced by the half-wave plate. As can be seen, a PC can introduce independent polarization direction rotation and polarization phase retardance to an incident light. In our system, polarimetric PT symmetry is achieved by controlling three critical angles in PC1 and PC2, which are listed in Table 1.

Specifically, the bending-induced birefringence of PC1 leads to a polarization phase retardance. Assuming that the fast and slow polarization components are $E_{x}$ and $E_{y}$, respectively, the phase retardance can be tuned by changing $\theta_{r}$ using the equivalent quarter-wave plates in PC1, which aligns the eigenmodes of the two polarimetric loops. The $m$-th order eigenmode of a loop resonator is given by

$$
\omega_{m}=\frac{(2 m \pi+\phi) c}{n_{e f f} L}
$$

where $\phi$ is the phase shift within the laser ring cavity, $c$ is the light velocity in a vacuum, and $n_{\text {eff }}$ and $L$ are the effective refractive index and the length of the optical fiber within the laser ring cavity, respectively. Although the physical lengths of the two polarimetric loops are the same, the eigenfrequencies of the cavities with the respective polarizations may be perturbed due to the residual birefringence originating from the fiber bending and the polarization mode dispersion (PMD) of the optical components, resulting in a mismatch of the eigenmodes between the two polarimetric cavities, as shown in Fig. 2a. Assuming that the birefringent phases of the two polarizations are $\phi_{x}$ and $\phi_{y}$, the separation between the localized eigenfrequencies is given by

$$
\delta \omega_{m}=\frac{\left(\phi_{x}-\phi_{y}\right) c}{n_{e f f} L}
$$

To achieve PT symmetry, it is required that the eigenfrequency separation is zero, which can be achieved by tuning the equivalent quarter-wave plate in $\mathrm{PC} 1$, i.e., $\theta_{r}$, to match the values of $\phi_{x}$ and $\phi_{y}$, thus aligning the 
eigenfrequencies of the polarimetric cavities for the implementation of PT symmetry between the real parts of the eigenfrequencies ${ }^{29}$.

On the other hand, the tuning of $\theta_{i}$ using the half-wave plate in $\mathrm{PC} 1$ can result in a rotation of the optical axis in the optical path from Pol. 1 to Pol. 2, which, in conjunction with the polarizers, adjusts the round-trip gain, loss, and coupling coefficients of the two polarimetric loops. PT symmetry between the imaginary parts of the eigenfrequencies of the two polarimetric loops can be achieved. We first assume that the polarization direction of the light is preserved from Pol. 2 to Pol. 1, i.e., $\theta_{t}=0$, and that the directions of Pol. 1 and Pol. 2 are aligned such that they are the same. The joint operation between Pol. 1 and Pol. 2 is then equivalent to a single polarizer. The relations between the electric fields at PC1 before and after one round trip in the cavity, denoted as $E^{(0)}$ and $E^{(1)}$, are given by

$$
\left[\begin{array}{l}
E_{x}^{(1)} \\
E_{y}^{(1)}
\end{array}\right]=\gamma_{0}\left[\begin{array}{ll}
\cos ^{2} \theta_{i} & \sin \theta_{i} \cos \theta_{i} \\
\sin \theta_{i} \cos \theta_{i} & \sin ^{2} \theta_{i}
\end{array}\right]\left[\begin{array}{c}
E_{x}^{(0)} \\
E_{y}^{(0)}
\end{array}\right]
$$

where $\gamma_{0}$ is the round-trip gain of the electric field of a polarization component when its polarization direction is perfectly aligned with that of the polarizer.

As the polarization components $E_{x}$ and $E_{y}$ recirculate in the fiber loop, their incidental angles respective to the principal axes of Pols. 1 and 2 result in round-trip gains, given by

$$
\begin{aligned}
& \gamma_{x}=\gamma_{0} \cos ^{2} \theta_{i} \\
& \gamma_{y}=\gamma_{0} \sin ^{2} \theta_{i}
\end{aligned}
$$

and a round-trip coupling strength given by

$$
\kappa=\gamma_{0} \sin \theta_{i} \cos \theta_{i}
$$

The round-trip gains $\gamma_{x}$ and $\gamma_{y}$ can be tuned continuously from 0 to a maximum value of $\gamma_{0}$. At PT symmetry, gain and loss balance should be achieved between the two polarimetric loops, i.e., $\gamma_{x} \gamma_{y}=1$. Based on Eq. (5), we find that the angle of polarization rotation for the implementation of PT symmetry for the imaginary part of the eigenfrequency is

$$
\theta_{\gamma}= \pm \frac{1}{2} \arcsin \left(\frac{2}{\gamma_{0}}\right)
$$

Last, the optical path from Pol. 2 to Pol. 1 is initially configured to be nonbirefringent by tuning $\mathrm{PC} 2$ to fully compensate for the polarization mode dispersion contributed by all optical components. The tuning of the equivalent half-wave plate in $\mathrm{PC} 2$ then introduces a propagation loss for any incident light to Pol. 1, as the polarization direction of light from Pol. 1 is no longer perfectly aligned with that of Pol. 2 . The maximum net round-trip gain becomes $\gamma_{0} \cos \theta_{t}$ instead of $\gamma_{0}$, where $\theta_{t}$ is the polarization rotation angle. Tuning $\theta_{t}$ is different from the case where $\theta_{i}$ is tuned. The tuning of $\theta_{i}$ introduces a variation in the gain difference between the polarimetric loops and can be used to achieve gain and loss balance. By contrast, the tuning of $\theta_{t}$ introduces a universal gain variation of $\cos \theta_{t}$ for both polarimetric loops and thus affects only the lasing threshold of the PT-symmetric laser. Tuning $\theta_{t}$ is an alternative way to adjust the lasing threshold, which is more precise and convenient than changing the pump current to the EDFA.

We then convert the round-trip gain, loss and coupling to the corresponding per-time-unit coefficients (see Supplementary Information 1), and the optical coupling between the two polarimetric loops can be written as (see Supplementary Information 2)

$$
\begin{aligned}
& \frac{d E_{x}}{d t}=-i \omega_{m} E_{x}+i \kappa E_{y}+\gamma_{x} E_{x} \\
& \frac{d E_{y}}{d t}=-i \omega_{m} E_{y}+i \kappa E_{x}+\gamma_{y} E_{y}
\end{aligned}
$$

where $\kappa$ is a real number ${ }^{30}$. The solution to the coupling equations shows that a PT-symmetric system has complex eigenfrequencies when the gain or loss coefficients have a higher magnitude than the coupling coefficient, in which the imaginary part represents the gain or loss coefficient of the mode ${ }^{14}$. When operating in PT symmetry, the system can provide a gain difference enhancement between the longitudinal mode with the highest roundtrip gain (dominant mode, $\gamma_{0}$ ) and that with the second highest gain (secondary mode, $\gamma_{1}$ ). The enhancement factor is given by

$$
F=\frac{\Delta g_{P T S}}{\Delta g_{\text {Hermitian }}}=\frac{\sqrt{\gamma_{0}^{2}-\gamma_{1}^{2}}}{\gamma_{0}-\gamma_{1}}
$$

where $\Delta g_{P T S}$ and $\Delta g_{\text {Hermitian }}$ are the gain differences between the dominant and secondary modes in a PTsymmetric system and a single-loop Hermitian system, respectively. The enhancement of the gain difference with a factor of $F$ would significantly reduce the difficulty in achieving stable single-mode lasing.

\section{Discussion}

An experiment is performed based on the setup shown in Fig. 1. Figure 3 shows the round-trip gain variations of the polarimetric loops measured by injecting two linearly and orthogonally polarized light waves into the open-loop 

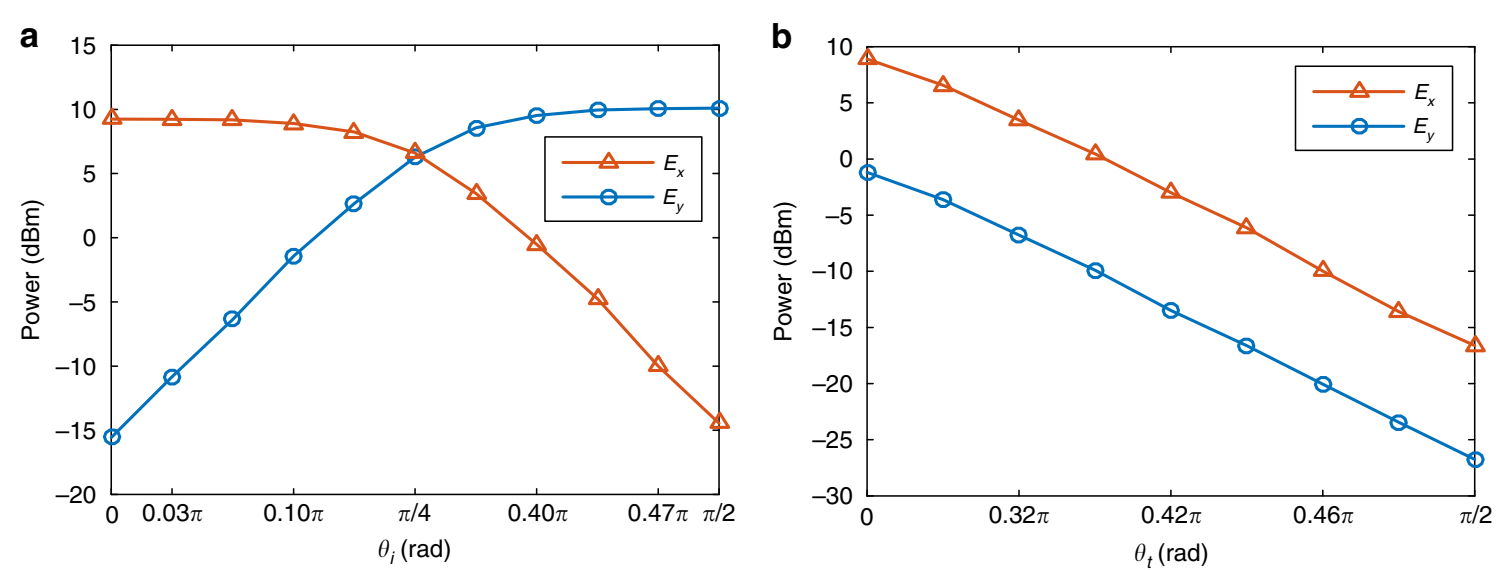

Fig. 3 Gain tuning in the two polarimetric loops achieved by tuning PC1 and PC2. The round-trip gain coefficients of the two polarimetric loops change $\mathbf{a}$ differentially and $\mathbf{b}$ uniformly when $\theta_{i}$ and $\theta_{t}$ are tuned, respectively. The measurement results agree well with the theoretical results shown in Fig. 2b, indicating that PT symmetry can be achieved by tuning PC1 and PC2

laser cavity. By tuning PC1 and PC2, the round-trip gain and gain difference between the two polarimetric loops are changed, which can be used to implement PT symmetry in the proposed polarimetric PT-symmetric fiber ring laser (see Supplementary Information 3).

To verify the PT symmetry operation of the fiber ring laser, the light at its output is directed to an optical spectrum analyzer for spectrum measurement, a homodyne system for longitudinal mode analysis, and a selfheterodyne system for spectral linewidth measurement. When the loop is closed and the EDFA operates with a sufficiently high gain to make the round-trip net gain exceed the loss, lasing begins. Figure 4a shows the homodyne spectrum. Since PC1 and PC2 are not tuned to achieve PT symmetry, multimode lasing with a mode spacing of $4.88 \mathrm{MHz}$ corresponding to a loop length of $41.0 \mathrm{~m}$ is observed from the measured electrical spectrum. As PC1 and PC2 are tuned to achieve PT symmetry, and to make the gain/loss greater than the coupling coefficient, PT symmetry is broken, and single-mode lasing is achieved. As can be seen in Fig. 4b, all the beat notes are suppressed except the one at DC, which clearly indicates that single-longitudinal-mode lasing is achieved. The measured optical spectrum is shown in Fig. 4c with a central wavelength of $1563.204 \mathrm{~nm}$. The highest beat notes from Fig. $4 \mathrm{a}, \mathrm{b}$ are suppressed by $47.9 \mathrm{~dB}$ compared to the DC, which indicates that a sidemode suppression ratio of approximately $47.9 \mathrm{~dB}$ is achieved due to the $\mathrm{PT}$ symmetry. The self-heterodyne spectrum is shown in the inset of Fig. 4b. A linewidth of approximately $129 \mathrm{kHz}$ is measured. By fitting the self-heterodyne spectrum to the Voigt profile ${ }^{31}$, we find that the Lorentzian linewidth $\Delta f_{L}$ and the Voigt linewidth $\Delta f_{V}$ are 2.4 and $128 \mathrm{kHz}$, respectively, which indicates that the light wave generated by the fiber ring laser has a very narrow intrinsic linewidth of $2.4 \mathrm{kHz}$ thanks to the long cavity length. On the other hand, the measured linewidth is $129 \mathrm{kHz}$, which is broadened due to the high susceptibility of the system to environmental disturbances because of a long fiber employed in the cavity. This broadening can be well compensated if active cavity stabilization or isolation is incorporated in the laser system ${ }^{31,32}$.

In addition, the power stability is also measured. As shown in the supplemental video, the power fluctuation is as low as $0.2 \mathrm{~dB}$ within a 30 -s measurement time frame. If the system is well packaged or active cavity stabilization or isolation is incorporated, the power stability can be further improved.

Then, the wavelength tunability of the fiber ring laser is studied. Figure 4c, d show the optical spectra of the light at the output of the fiber ring laser when the wavelength is tuned with a minimum tuning step of $50 \mathrm{pm}$ and a maximum tuning range of $25 \mathrm{~nm}$, from 1540 to $1565 \mathrm{~nm}$. This is achieved by tuning the TOF, which has a bandwidth of $0.1 \mathrm{~nm}$, within which there are approximately 2016 longitudinal modes. PT symmetry is then realized to help select the longitudinal mode with the highest roundtrip gain from the 2,016 longitudinal modes to enable stable single-mode lasing.

The threshold behavior of the fiber ring laser is also studied. Figure 5a shows the optical spectrum at the output of the fiber ring laser. As shown, when the gain is below the threshold, the fiber ring laser is in the nonlasing mode, and a broad spectrum, which is the filtered amplified spontaneous emission (ASE), is produced. When the gain is sufficiently high as a result of reducing the intracavity loss by tuning $\mathrm{PC} 2$, lasing starts, and a high-power optical output at $1562.712 \mathrm{~nm}$ is observed. Figure $5 \mathrm{~b}$ shows the output power when the intracavity loss is tuned. 

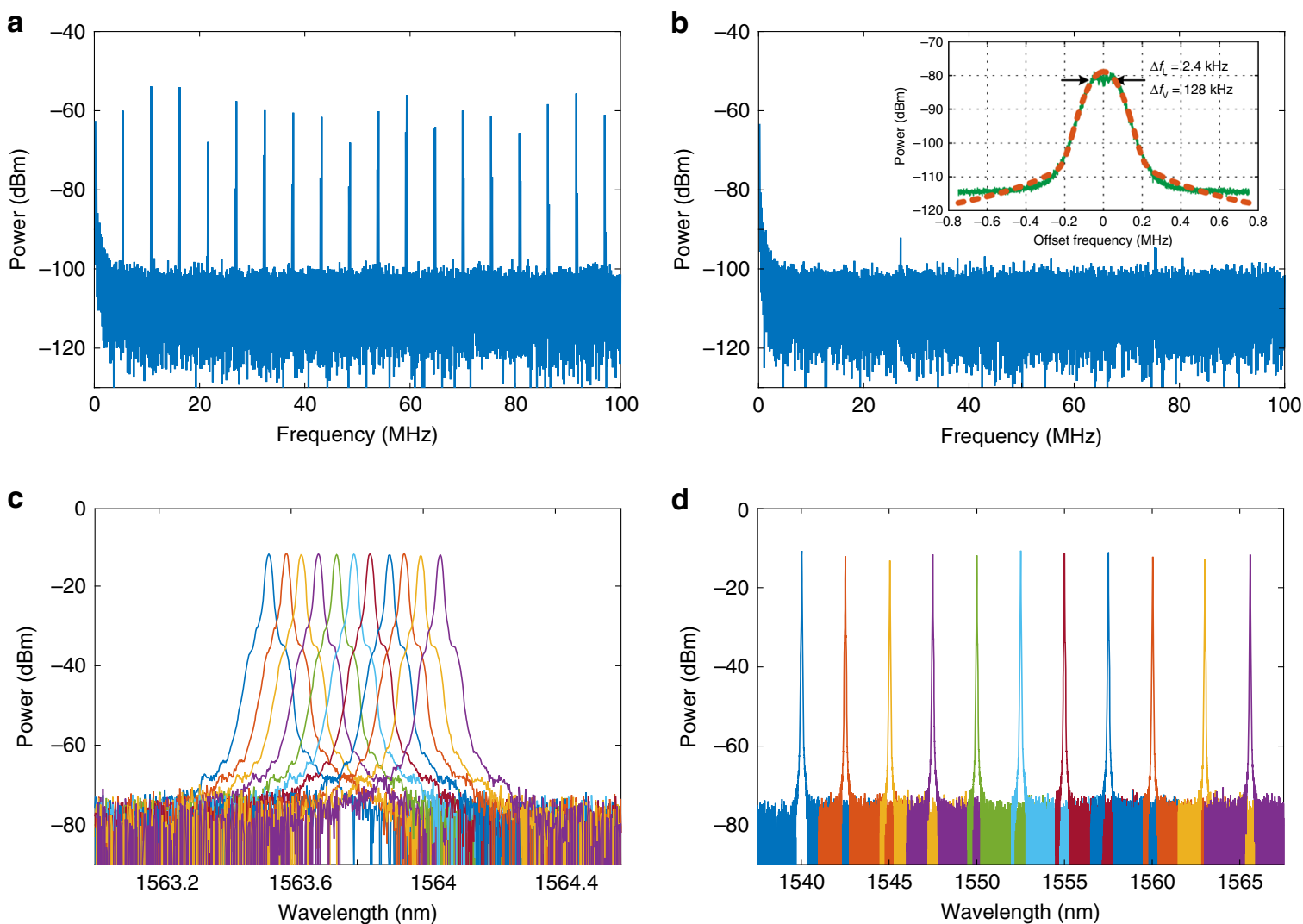

Fig. 4 Spectrum analysis. a The homodyne spectrum when the system is operating without PT symmetry. Multimode oscillation is observed. $\mathbf{b}$ The homodyne spectrum when the system is operating with PT symmetry by tuning PC1 and PC2. Single-mode oscillation is observed. The inset shows that the laser linewidth is measured to be $129 \mathrm{kHz}$, which is broadened due to the environmental perturbations in the long-cavity laser. The Lorentzian linewidth is calculated to be $2.4 \mathrm{kHz}$. c, $\mathbf{d}$ The optical spectra of the single-mode laser when the wavelength is $\mathbf{c}$ fine-tuned with a step of $50 \mathrm{pm}$ from 1563.55 to $1564.05 \mathrm{~nm}$ and $\mathbf{d}$ coarsely tuned with a step of $2.5 \mathrm{~nm}$ from 1540 to $1565 \mathrm{~nm}$
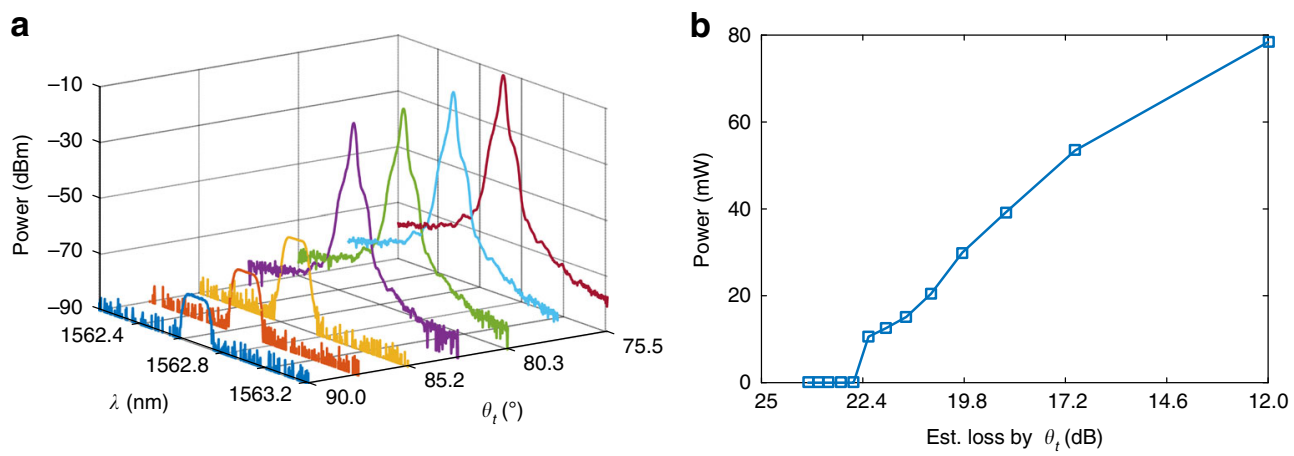

Fig. 5 Optical spectrum and output power of the fiber ring laser when the cavity loss is tuned by tuning PC2. a Optical spectrum evolution of the laser output when the intracavity loss is tuned by tuning PC2, showing the threshold-like behavior of the polarimetrically PT-symmetric laser. b Output optical power measured from the optical spectrum at peak wavelength when the intracavity loss is tuned within a range of $10 \mathrm{~dB}$. Note that an optical attenuator is incorporated when the optical spectrum is measured to avoid high-power damage to the OSA

In conclusion, we have proposed and demonstrated that PT symmetry can be implemented between two subspaces in a single spatial unit based on polarimetric diversity. By controlling the polarization states of light in the single spatial unit, the localized eigenfrequencies, gain, loss, and coupling coefficients could be tuned, leading to PT 
symmetry breaking. The polarimetric PT symmetry was experimentally verified by a fiber ring laser in which a single physical loop supporting two mutually coupled polarimetric loops was implemented. Stable singlelongitudinal-mode lasing was achieved. The linewidth of the light generated by the laser was measured to be $129 \mathrm{kHz}$ for a wavelength tuning range of $35 \mathrm{~nm}$. With active stabilization implemented in the fiber laser, the linewidth could be reduced to its Lorentzian linewidth of $2.4 \mathrm{kHz}$. Since only a single physical loop is required, the implementation is significantly simplified, and the stability is highly improved.

\section{Materials and methods}

The polarimetric PT symmetry is demonstrated based on a fiber ring laser implemented using commercial offthe-shelf optical components. The EDFA (Amonics AEDFA-27-B-FA) has an operating wavelength range from 1535 to $1565 \mathrm{~nm}$ and a fixed output power of $27 \mathrm{dBm}$. The TOF (Alnair Labs BVF-300CL) has a tunable 3-dB bandwidth from 0.03 to $3 \mathrm{~nm}$ and a tunable central wavelength from 1525 to $1610 \mathrm{~nm}$. The PCs are Thorlabs FPC032 manual paddle fiber polarization controllers. The polarizers are implemented with a polarization beam combiner/splitter (Thorlabs PBC1550SM-APC) with only two reciprocally transmitting ports.

The performance of the system is evaluated using benchtop instruments. The optical spectra are measured with an OSA (Yokogawa AQ6370C). An optical homodyne is used for laser modal analysis due to its high dynamic range, which is realized by directly launching the light at the output of the laser to a PD (photodetector, Discovery Semiconductor DSC20H) and measuring the electrical spectrum at the output of the PD with an ESA (electrical spectrum analyzer, Keysight N9020B). The linewidth of the light wave generated by the laser is measured based on the optical self-heterodyne, in which a 40-km-long single-mode fiber is used to introduce a long time delay and an intensity modulator (Photline MX-LN40), biased at the minimum transmission point driven by a microwave source (HMC-T2220), is used to slightly shift the wavelength to make the beat note easier to observe. The optical self-heterodyne spectrum is also measured with a PD (Discovery Semiconductor DSC20H) and an ESA (Keysight N9020B).

\section{Acknowledgements}

This work is supported by the National Natural Science Foundation of China (61860206002, 61905095, 61805103).

\section{Author contributions}

L.L., Y.C. and Y. Zhi contributed equally to the paper; J.Z. conceived the idea, designed the experiment, and wrote the paper; L.L., Y.C., Y. Zhi and Y. Zou performed the experiment, analyzed the data, and wrote the paper; J.Y. analyzed the data and wrote the paper; and X.F. and B.G. wrote the paper.
Conflict of interest

The authors declare that they have no conflict of interest.

Supplementary information is available for this paper at https://doi.org/ 10.1038/s41377-020-00407-3.

Received: 30 July 2020 Revised: 9 September 2020 Accepted: 10 September 2020

Published online: 27 September 2020

\section{References}

1. Regensburger, A. et al. Parity-time synthetic photonic lattices. Nature $\mathbf{4 8 8}$, 167-171 (2012).

2. Peng, B. et al. Parity-time-symmetric whispering-gallery microcavities. Nat. Phys. 10, 394-398 (2014)

3. Hodaei, $\mathrm{H}$. et al. Enhanced sensitivity at higher-order exceptional points. Nature 548, 187-191 (2017).

4. Perez-Leija, A. et al. Coherent quantum transport in photonic lattices. Phys. Rev. A 87, 012309 (2013)

5. Hodaei, H. et al. Parity-time-symmetric microring lasers. Science 346, 975-978 (2014).

6. Feng, L. et al. Single-mode laser by parity-time symmetry breaking. Science 346, 972-975 (2014).

7. Wong, Z. J. et al. Lasing and anti-lasing in a single cavity. Nat. Photon. $\mathbf{1 0}$ 796-801 (2016).

8. Wan, W. J. et al. Time-reversed lasing and interferometric control of absorption. Science 331, 889-892 (2011).

9. Zhu, X. F. et al. One-way invisible cloak using parity-time symmetric transformation optics. Opt. Lett. 38, 2821-2824 (2013).

10. Sounas, D. L., Fleury, R. \& Alù, A. Unidirectional cloaking based on metasurfaces with balanced loss and gain. Phys. Rev. Appl. 4, 014005 (2015).

11. El-Ganainy, R. et al. Non-Hermitian physics and PT symmetry. Nat. Phys. 14 11-19 (2018).

12. Özdemir, S. K. et al. Parity-time symmetry and exceptional points in photonics. Nat. Mater. 18, 783-798 (2019)

13. Feng, L., El-Ganainy, R. \& Ge, L. Non-Hermitian photonics based on parity-time symmetry. Nat. Photon. 11, 752-762 (2017).

14. Zhang, J. J. \& Yao, J. P. Parity-time-symmetric optoelectronic oscillator. Sci. Adv. 4, eaar6782 (2018)

15. Liu, Y. Z. et al. Observation of parity-time symmetry in microwave photonics. Light Sci. Appl. 7, 38 (2018).

16. Schawlow, A. L. \& Townes, C. H. Infrared and optical masers. Phys. Rev. 112 1940-1949 (1958).

17. Beier, F. et al. Narrow linewidth, single mode $3 \mathrm{~kW}$ average power from a directly diode pumped ytterbium-doped low NA fiber amplifier. Opt. Express 24, 6011-6020 (2016)

18. $\mathrm{Ma}$, R. L. et al. Tunable sub-kHz single-mode fiber laser based on a hybrid microbottle resonator. Opt. Letters 43, 5315-5318 (2018).

19. Mehravar, S. et al. Real-time dual-comb spectroscopy with a free-running bidirectionally mode-locked fiber laser. Appl. Phys. Lett. 108, 231104 (2016).

20. Quintino, L. et al. Welding with high power fiber lasers-A preliminary study. Mater. Des. 28, 1231-1237 (2007).

21. Richardson, D. J., Nilsson, J. \& Clarkson, W. A. High power fiber lasers: current status and future perspectives [Invited]. J. Opt. Soc. Am. B 27, B63-B92 (2010).

22. Chen, M. et al. Ultra-narrow-linewidth brillouin/erbium fiber laser. CLEO QELS_Fundamental Science (OSA, San Jose, California, 2017).

23. Zhou, K. J. et al. kHz-order linewidth controllable $1550 \mathrm{~nm}$ single-frequency fiber laser for coherent optical communication. Opt. Express 25, 19752-19759 (2017).

24. Zhang, J. J. et al. Microfiber Fabry-Perot interferometer fabricated by taperdrawing technique and its application as a radio frequency interrogated refractive index sensor. Opt. Lett. 37, 2925-2927 (2012).

25. Meng, Z., Stewart, G. \& Whitenett, G. Stable single-mode operation of a narrow-linewidth, linearly polarized, erbium-fiber ring laser using a saturable absorber. J. Lightwave Technol. 24, 2179-2183 (2006).

26. Chen, X. F. et al. Single-longitudinal-mode fiber ring laser employing an equivalent phase-shifted fiber Bragg grating. IEEE Photon. Technol. Lett. 17, 1390-1392 (2005). 
27. Zhang, J. J. et al. Parity-time symmetry in wavelength space within a single spatial resonator. Nat. Commun. 11, 3217 (2020).

28. Liu, W. L., Wang, M. G. \& Yao, J. P. Tunable microwave and sub-terahertz generation based on frequency quadrupling using a single polarization modulator. J. Lightwave Technol. 31, 1636-1644 (2013).

29. Ulrich, R., Rashleigh, S. C. \& Eickhoff, W. Bending-induced birefringence in single-mode fibers. Opt. Lett. 5, 273-275 (1980).
30. Hui, R. Q. \& O'Sullivan, M. Fiber Optic Measurement Techniques (Academic Press, San Diego, 2009).

31. Viciani, S. et al. Lineshape of a vertical cavity surface emitting laser. Opt. Commun. 206, 89-97 (2002).

32. Li, Y. H. et al. Photonic generation of W-band arbitrary waveforms with high time-bandwidth products enabling $3.9 \mathrm{~mm}$ range resolution. Optica 1 446-454 (2014) 